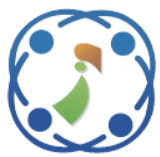

\title{
TIMBO: Three Influential Members Based Optimizer
}

\author{
Fatemeh Ahmadi Zeidabadi ${ }^{1}$ \\ Mohammad Dehghani ${ }^{2 *}$ \\ Om Parkash Malik ${ }^{3}$ \\ ${ }^{1}$ Department of Mathematics and Computer Sciences, Sirjan University of Technology, Sirjan, Iran \\ ${ }^{2}$ Graduate of the Dept. of Electrical and Electronics Engineering, Shiraz University of Technology, Shiraz, Iran \\ ${ }^{3}$ Department of Electrical Engineering, University of Calgary, Calgary Alberta Canada \\ * Corresponding author's Email: adanbax@gmail.com
}

\begin{abstract}
One of the most important and efficient methods in providing suitable solutions for various optimization problems is population-based optimization algorithms. The main contribution and innovation of this paper is to present a new optimization method called Three Influential Members Based Optimizer (TIMBO) which is used for implementation in solving optimization problems. The main idea in designing the proposed TIMBO is to use three important population members with the titles of best member, worst member, and member as mean population in updating the position of population members of the algorithm in the problem search space. The most important feature and advantage of the TIMBO is that it does not have any control parameters, which means that there is no need to control the parameter in this algorithm. TIMBO has been mathematically modeled for use in solving various optimization problems. The efficiency of the TIMBO is analyzed in order to provide suitable quasi-optimal solutions on a set of twenty-three standard objective functions of different types unimodal, high-dimensional multimodal, and fixed-dimensional. Evaluation of unimodal functions indicates the high exploitation power of the proposed TIMBO and evaluation of multimodal functions indicates the appropriate exploration power of the TIMBO. Also, the results obtained from the TIMBO are compared with the performance of eight other well-known optimization algorithms including Genetic Algorithm (GA), Particle Swarm Optimization (PSO), Gravitational Search Algorithm (GSA), Teaching-Learning-Based Optimization (TLBO), Grey Wolf Optimization (GWO), Grasshopper Optimisation Algorithm (GOA), Hide Object Game Optimizer (HOGO), and Flow Direction Algorithm (FDA). The results of optimization of standard objective functions indicate the high capability of the TIMBO in providing quasi-optimal solutions suitable for various optimization problems. In addition, analyzing and comparing the performance of the other eight optimization algorithms shows that the TIMBO has a more effective ability to solve optimization problems and is much more competitive.
\end{abstract}

Keywords: Optimization, Population-based, Optimization problems, Best member, Worst member, Mean member.

\section{Introduction}

Optimization means finding the best solution out of all the possible solutions to a problem given its limitations. An optimization problem is defined using three main parts: variables, constraints, and objective functions. The goal in optimization is to determine the values of the variables according to the constraints in order to optimize the objective functions [1]. Population-based optimization algorithms are one of the most widely used methods in solving optimization problems that search the problem-solving space using random operators [2].
The main solution to an optimization problem is called global optimal. The important thing about optimization algorithms is that the solutions obtained from them are not necessarily global optimal. For this reason, the solution provided by the optimization algorithms is called quasi-optimal solution. In other words, a quasi-optimal solution is a solution that, if not equal to the global optimal, must be reasonably close to it [3]. Therefore, in comparing the performance of optimization algorithms with each other, the algorithm that provides a better quasioptimal solution is a better algorithm. This is the reason why researchers have designed various 
optimization algorithms.

The contribution of this paper is to design a new population-based optimization algorithm called Three Influential Members Based Optimizer (TIMBO) in order to provide suitable and nearoptimal quasi-optimal solutions. Updating population members is influenced by three members named best member, worst member, and mean member is the main idea in designing the TIMBO. The proposed TIMBO is mathematically modeled for use in solving optimization problems in various science. The main advantage and feature of the TIMBO are that it lacks control parameters and does not need to be adjusted. The performance of the TIMBO is evaluated on a set of twenty-three standard objective functions, then the results are compared with eight other optimization algorithms.

The rest of the paper is as follows: First, literature review is provided in Section 2. Then in section 3 the proposed TIMBO is introduced. Simulation studies and analysis of results is presented in Section 4. Finally, conclusions and suggestions for future studies are provided in Section 5.

\section{Lecture review}

Optimization has been one of the most important research areas in recent decades, which has led to the design of various types of population-based optimization algorithms. In population-based methods, search agents work together and the collective behaviour of these agents causes the algorithm to converge toward a solution to the optimization problem [4]. Optimization algorithms are based on observations and thinking about natural events, physical phenomena, the behavior of living things, various games, and any other process that has an evolutionary context [5].

Genetic Algorithm (GA) is a probabilistic optimization algorithm with a global search potential which is proposed by Holland in 1975. GA is one of the most successful evolutionary algorithms. GA is developed based on the modeling of the reproductive process in three stages: parental selection, crossover, and mutation [6]. The main disadvantage of GA is that if the objective function is not well defined, GA may select the local optimal instead of the global optimal.

Particle Swarm Optimization (PSO) is a nationwide population-based optimization technique which is proposed by Kennedy and Eberhart in 1995. This method is inspired by the social behavior of birds in search of food. Due to its simple search mechanism, computational efficiency and easy implementation, PSO is widely used in many areas of optimization [7]. The main disadvantage of the PSO is that has several control parameters, and setting these parameters is an important challenge.

Gravitational Search Algorithm (GSA) is a physics-based optimization algorithm which is developed by Rashedi in 2009. In GSA, search agents are considered as objects and their efficiency is considered as their mass. All of these objects absorb each other with a gravitational force that causes all objects to have a general motion toward objects that have a greater mass than other objects. So, objects work together to achieve better solutions through a direct connection that is gravitational force [8]. High computations as well as long time required to run are the main disadvantages of GSA.

Teaching-Learning-Based Optimization (TLBO) algorithm is one of the intelligent optimization algorithms and crowded intelligence that was introduced by Rao in 2011 inspired by the learning and teaching process. TLBO is a population-based algorithm based on the impact of a teacher on classroom learning. Population is considered as a group of learners or students of a class. A teacher strives to increase the level of knowledge in the classroom by educating students so that students can achieve a good grade or rank according to their ability [9]. low performance in the convergence rate is a major disadvantage of TLBO which gets even worse when dealt with high-dimensional optimization problems.

Grey Wolf Optimization (GWO) algorithm which mimics the grey wolves' behaviors for hunting in the nature, is introduced by Mirjalili in 2014. GWO is a metaheuristic algorithm inspired by the hierarchical structure and social behavior of grey wolves while hunting. This population-based algorithm has a simple process and can be easily generalized to largescale problems [10]. The main disadvantages of GWO are low solving precision, slow convergence, and bad local searching ability.

Grasshopper Optimisation Algorithm (GOA) is a swarm-based optimization algorithm which is introduced by Saremi in 2017. GOA belongs to the group of collective intelligence algorithms and is designed based on the social behaviour of grasshoppers and how each grasshopper is affected by its environment. In GOA, updating the position of each grasshopper depends on the distance of each locust from the entire population of grasshoppers in the current generation and the position of the best grasshopper [11]. GOA has some disadvantages, such as easy to fall into local optimum and slow convergence speed.

Hide Object Game Optimizer (HOGO) is a gamebased optimization approach which is developed by 
Dehghani in 2020. HOGO is designed based on simulation of the rules and behavior of players in a game called finding hidden object. The search agents are the players of this game who try to find the hidden object, which is the solution to the problem, by moving in the problem-solving space [12]. Although HOGO performs well in solving optimization problems, the main challenge of this algorithm is to find more suitable and quasi-optimal solutions closer to the global optimization.

Flow Direction Algorithm (FDA) is a physicsbased optimization algorithm which is presented by Karami in 2021. FDA is designed based on mimic of the flow direction to the outlet point with the lowest height in a drainage basin. In other words, flow moves to neighbour with lowest high or best objective function [13]. The main disadvantages of the FDA are slow convergence and also having several control parameters that need to be fine-tuned. In fact, if the parameters of the FDA are not set well, the algorithm diverges and is not able to achieve the appropriate solution.

Although disadvantages such as having control parameters, time consuming, and high computational volume can affect the performance of optimization algorithms, but the most important criterion in analyzing the performance of optimization algorithms is the value of the objective function. In fact, in comparing and analyzing the performance of optimization algorithms on solving an optimization problem, the algorithm that can provide a more suitable quasi-optimal solution is the superiority algorithm.

\section{Three influential members based optimizer}

The various steps and mathematical modelling of proposed Three Influential Members Based Optimizer (TIMBO) are presented in this section. TIMBO is a population-based optimization algorithm that first generates a number of initial solutions to an optimization problem, then improves these initial solutions in an iterative process, and finally presents the best obtained solution. The main idea of the proposed TIMBO is to improve and update the members of the population under the influence of three important members of the population: the best member, the worst member, and the mean member.

The population members in the TIMBO are modeled as a matrix representation using Eq. (1).

$$
X=\left[\begin{array}{c}
X_{1} \\
\vdots \\
X_{i} \\
\vdots \\
X_{N}
\end{array}\right]_{N \times m}=\left[\begin{array}{ccccc}
x_{1,1} & \cdots & x_{1, d} & \cdots & x_{1, m} \\
\vdots & \ddots & \vdots & \ddots & \vdots \\
x_{i, 1} & \cdots & x_{i, d} & \cdots & x_{i, m} \\
\vdots & \ddots & \vdots & \ddots & \vdots \\
x_{N, 1} & \cdots & x_{N, d} & \cdots & x_{N, m}
\end{array}\right]_{N \times m}
$$

Here, $X$ is the population matrix of TIMBO, $X_{i}$ is the $i$ th member of population, $m$ is the number of variables of optimization problem, $N$ is the number of members of population, and $x_{i, d}$ is the value of $d$ 'th variable of optimization problem suggested by $i$ 'th member of population.

In TIMBO, each member of the population represents a solution to the problem. In fact, each member of the population proposes values for the problem variables. By placing these values in the objective function, a value for the objective function is obtained in proportion to each member of the population. Thus, the objective function vector is modelled using Eq. (2).

$$
F=\left[\begin{array}{c}
F_{1} \\
\vdots \\
F_{i} \\
\vdots \\
F_{N}
\end{array}\right]_{N \times 1}=\left[\begin{array}{c}
F_{1}\left(X_{1}\right) \\
\vdots \\
F_{i}\left(X_{i}\right) \\
\vdots \\
F_{N}\left(X_{N}\right)
\end{array}\right]_{N \times 1}
$$

Here, $F$ is the vector of objective function and $F_{i}$ is the obtained value for objective function based on $i$ 'th member of population.

After evaluating the objective function based on population members, the best member and the worst member are determined by comparing the values of the objective function. The mean member is also obtained from the average of the population. These three influential members on population updating are identified using Eqs. (3) to (5).

$X^{B}=X_{j} \& j$ is the row number of $\min F$

$X^{W}=X_{k} \& k$ is the row number of $\max F$

$$
X^{M}: x_{d}^{M}=\frac{\sum_{i=1}^{N} x_{i, d}}{N}
$$

Here, $X^{B}$ is the best member, $X^{W}$ is the worst member, and $X^{M}$ is the mean member. 
In propoed TIMBO each member of the population is updated in three stages. In the first stage, each member of the population is updated based on the best member of the population, which is modeled using Eqs. (6) to (7).

$$
\begin{aligned}
& x_{i, d}^{B, \text { new }}=x_{i, d}+r\left(x_{d}^{B}-x_{i, d}\right) \\
& X_{i}=\left\{\begin{aligned}
X_{i}^{B, \text { new }}, & F_{i}^{B, \text { new }}<F_{i} \\
X_{i}, & \text { else }
\end{aligned}\right.
\end{aligned}
$$

Here, $x_{i, d}^{B, \text { new }}$ is the new value suggested by $i^{\prime}$ th member for $d$ 'th variable updated based on best member, $x_{d}^{B}$ is the value of $d^{\prime}$ th variable suggested by best member, $r$ is a random number in [0 1$]$ interval, $X_{i}^{B, \text { new }}$ is the updated $i$ th member based on best member, and $F_{i}^{B, n e w}$ is the its objective function value.

In the second stage of population updating, population members move under the influence of the worst member in the problem-solving space. This stage is modeled using Eqs. (8) to (9).

$$
x_{i, d}^{W, \text { new }}=x_{i, d}+r\left(x_{i, d}-x_{d}^{W}\right)
$$

$$
X_{i}=\left\{\begin{aligned}
X_{i}^{W, \text { new }}, & F_{i}^{W, \text { new }}<F_{i} \\
X_{i}, & \text { else }
\end{aligned}\right.
$$

Here, $x_{i, d}^{W, n e w}$ is the new value suggested by $i$ th member for $d$ 'th variable updated based on worst member, $x_{d}^{W}$ is the value of $d^{\prime}$ th variable suggested by worst member, $X_{i}^{W, \text { new }}$ is the updated $i$ 'th member based on worst member, and $F_{i}^{W, n e w}$ is the its objective function value.

In the third stage, each member of the population is updated under the influence of the mean member. This stage of population updating is modeled using Eqs. (10) to (12).

$$
d x_{i, d}^{M}= \begin{cases}r\left(x_{d}^{M}-x_{i, d}\right), & F^{M}<F_{i} \\ r\left(x_{i, d}-x_{d}^{M}\right), & \text { else }\end{cases}
$$

$$
x_{i, d}^{M, n e w}=x_{i, d}+d x_{i, d}^{M}
$$

$$
X_{i}=\left\{\begin{aligned}
X_{i}^{M, n e w}, & F_{i}^{M, \text { new }}<F_{i} \\
X_{i}, & \text { else }
\end{aligned}\right.
$$

Here, $d x_{i, d}^{M}$ is the amount of changes for $d^{\prime}$ th variables suggested by $i$ 'th member, $F^{M}$ is the value of objective function for mean member, $x_{i, d}^{M, n e w}$ is the new value suggested by $i$ 'th member for $d$ 'th variable updated based on mean member, $X_{i}^{M, n e w}$ is the updated $i$ th member based on mean member, and $F_{i}^{M, n e w}$ is the its objective function value.

After all members of the population have been updated, the new values obtained for the problem variables are evaluated in the objective function, then the three affected members are updated and the members of the population are updated again based on these three updated members. In fact, the algorithm is repeated according to Eqs. (3) to (12) until the end of the algorithm. After completing the iterations of the algorithm, TIMBO provides the best quasi-optimal solution available. The various stages of TIMBO are presented as a flowchart in Fig. 1.

\section{Simulation study and discussion}

In this section, the simulation studies of the proposed TIMBO and its implementation on various optimization problems are discussed. The performance of the TIMBO in providing optimal quasi-optimal solutions is evaluated on twenty-three standard objective functions of unimodal, highdimensional multi-model, and fixed- dimensional multi-model types. Eight well-known optimization algorithms including Genetic Algorithm (GA) [6], Particle Swarm Optimization (PSO) [7], Gravitational Search Algorithm (GSA) [8], Teaching-Learning-Based Optimization (TLBO) [9], Grey Wolf Optimization (GWO) [10], Grasshopper Optimisation Algorithm (GOA) [11], Hide Object Game Optimizer (HOGO) [12], and Flow Direction Algorithm (FDA) [13] are intended to compare the results obtained from the implementation of the TIMBO. The performance results of the optimization algorithms are reported using the two indices of the average of best quasi-optimal solution (ave) and their standard deviation (std).

In order to evaluate the proposed TIMBO in solving the unimodal problems, seven objective 

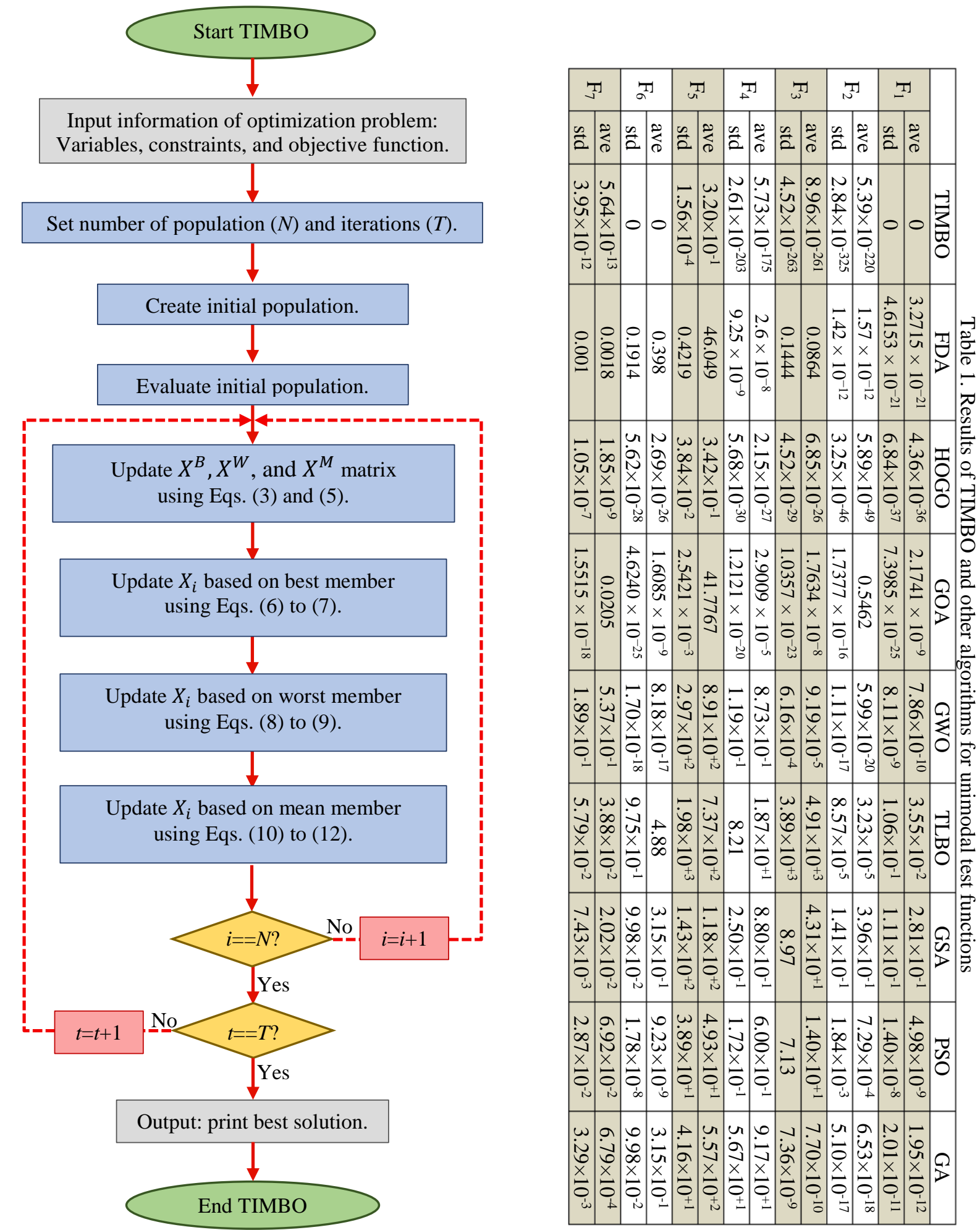

Figure. 1 Flowchart of TIMBO

functions F1 to F7 have been selected. The results of optimization of these objective functions using the TIMBO as well as eight other algorithms are given in Table 1 Analysis and comparison of the results of optimization algorithms show that the proposed TIMBO, in addition to providing good performance in solving these problems, is also superior to other algorithms. Six objective functions including F8 to F13 have been selected to analyse the ability of the TIMBO to solve high-dimensional multi-model optimization problems. The performance of the optimization algorithms and the TIMBO on these 


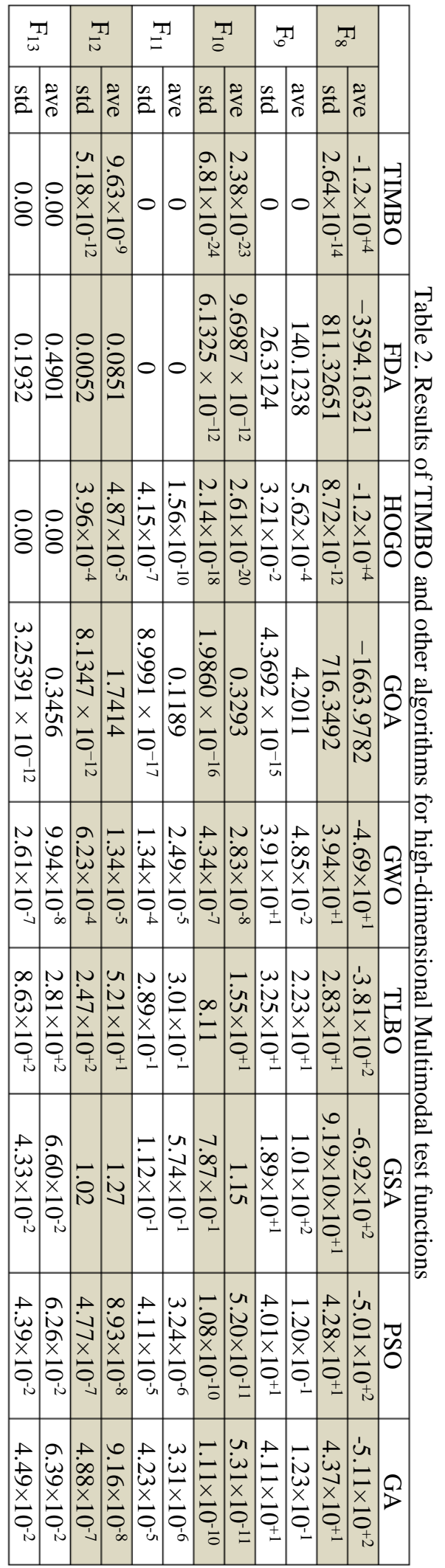

\begin{tabular}{|c|c|c|c|c|c|c|c|c|c|c|c|}
\hline W & N & $\stackrel{N}{N}^{T}$ & ${ }_{0}^{\top N}$ & $\frac{\pi}{6}$ & & $\underset{\infty}{T}$ & $\underset{\pi}{\pi}$ & $\frac{\pi}{\sigma}$ & $\frac{\pi}{n}$ & & 5 \\
\hline 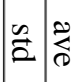 & $\begin{array}{c}2 \\
\text { ât }\end{array}$ & $\frac{\tilde{L}}{2} \mid \frac{\tilde{z}}{2}$ & 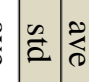 & $\frac{n}{2}$ & & $\frac{1}{2} \int_{0}^{2}$ & 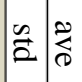 & $\mid \begin{array}{l}n \\
\vec{D}\end{array}$ & 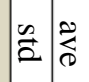 & & $\tilde{\check{c}}$ \\
\hline 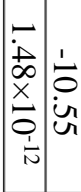 & 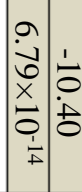 & 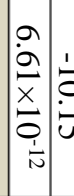 & 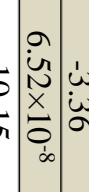 & 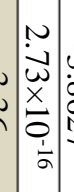 & 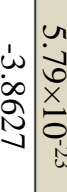 & 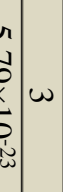 & 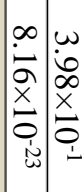 & 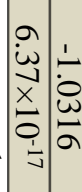 & 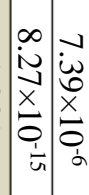 & & $\begin{array}{l}0 \\
\dot{0} \\
\infty \\
\infty\end{array}$ \\
\hline
\end{tabular}

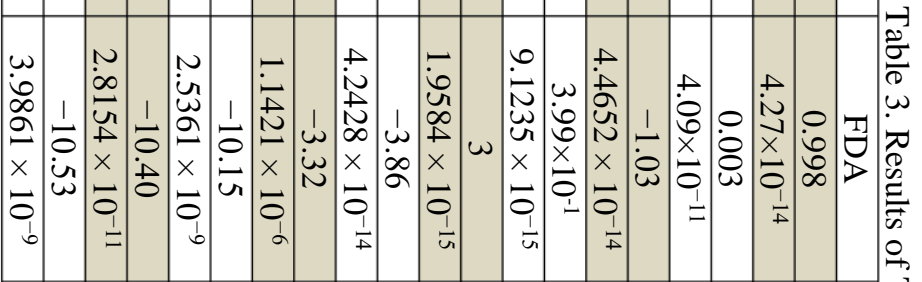

N w

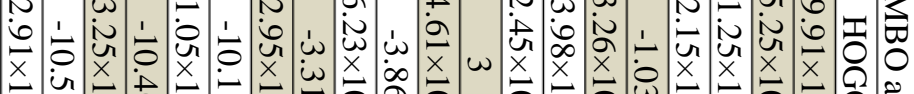

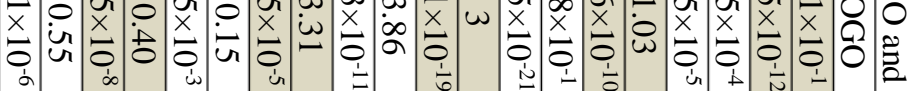

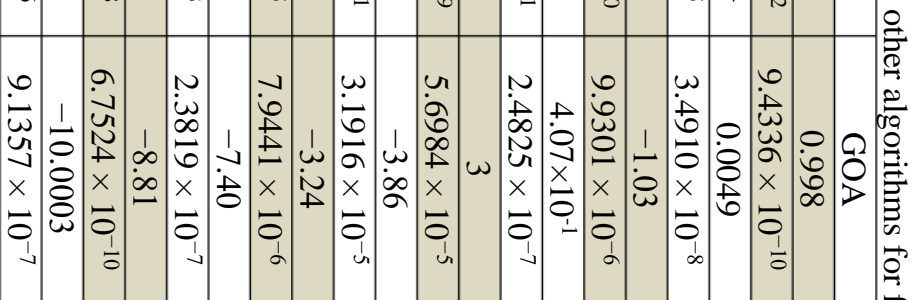

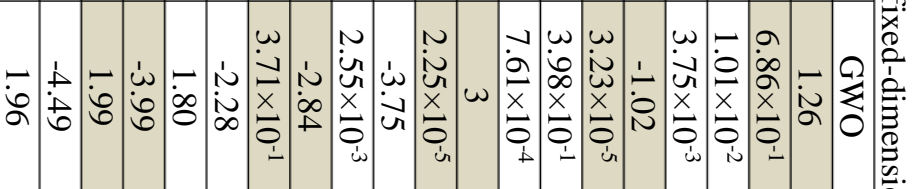

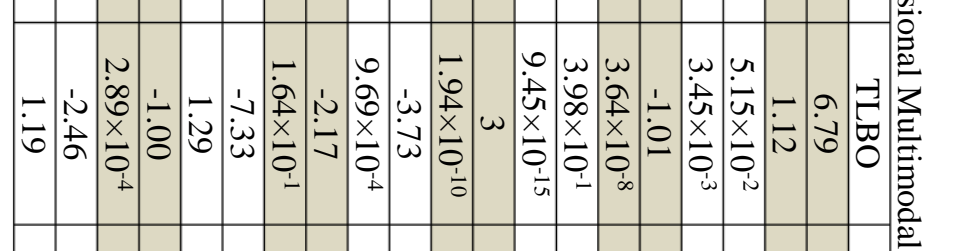

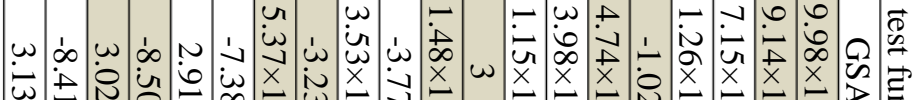

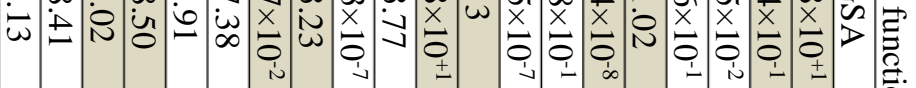

\begin{tabular}{|c|c|c|c|c|c|c|c|c|c|c|c|c|c|c|c|}
\hline J & $\infty$ & ما & $\stackrel{\sim}{\stackrel{N}{v}}$ & 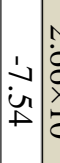 & & 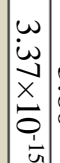 & 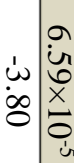 & & $\begin{array}{l}0 \\
0 \\
\dot{\omega} \\
x \\
\vdots \\
\vdots \\
\vdots \\
\sigma\end{array}$ & & กิ & $\begin{array}{l}\infty \\
x \\
0 \\
0\end{array}$ & $\begin{array}{c}\mathbf{x} \\
\mathbf{x} \\
\dot{w}\end{array}$ & & 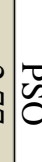 \\
\hline & 5 & $\rho$ & $\vec{\Delta}$ & 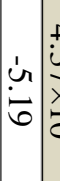 & 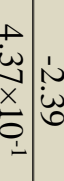 & 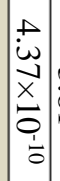 & 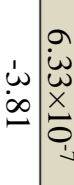 & 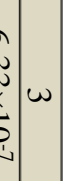 & 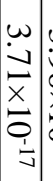 & $\begin{array}{l}0 \\
0 \\
0 \\
2 \\
0 \\
2 \\
\end{array}$ & 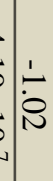 & & 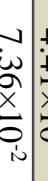 & & \\
\hline
\end{tabular}

functions are presented in Table 2 Based on the results reported in this table, TIMBO provides more suitable quasi-optimal solutions than the eight mentioned algorithms. 
In order to analyse the TIMBO in solving fixeddimensional multi-model optimization problems, ten objective functions F14 to F23 are considered. The results of the implementation of the proposed TIMBO and eight other algorithms are presented in Table 3 TIMBO has a good performance in solving this type of optimization problems.

\section{Conclusion and future works}

There are many optimization problems in different sciences that should be optimized and solved using appropriate methods. population based optimization algorithms are one of the most widely used methods in this field, which provide appropriate solutions to the problem based on random search in the problem-solving space. In this paper, in order to find suitable quasi-optimal solutions for various optimization problems, a new optimization algorithm called Three Influential Members Based Optimizer (TIMBO) was designed. Using three influential members named best member, worst member, mean member in updating the algorithm population was the main idea in designing the TIMBO. Mathematical Modelling of the TIMBO that can be used to solve optimization problems was presented. The quality of the proposed TIMBO was tested on twenty-three standard objective functions of different types. These objective functions come from three different groups including seven unimodal functions, six multimodal high-dimensional functions, and ten fixeddimensional multimodal functions. The results of optimization of unimodal objective functions showed that the proposed TIMBO has a high ability to provide quasi-optimal solutions suitable for optimization problems with unimodal objective functions. Also, the optimization results of highdimensional multimodal and fixed-dimensional multimodal objective functions indicated that the TIMBO can provide solutions close to the global optimal by accurately scanning the search space. The results of optimization of these objective functions indicated the optimal performance of the TIMBO in providing quasi-optimal solutions in solving various optimization problems with different objective functions. Also, in order to compare the performance of the proposed TIMBO, the results were compared with eight other optimization algorithms, including Genetic Algorithm (GA), Particle Swarm Optimization (PSO), Gravitational Search Algorithm (GSA), Teaching-Learning-Based Optimization (TLBO), Grey Wolf Optimization (GWO), Grasshopper Optimisation Algorithm (GOA), Hide Object Game Optimizer (HOGO), and Flow Direction Algorithm (FDA). The simulation results showed that the TIMBO has high power in solving optimization problems and is much more competitive than the other eight known algorithms.

The authors suggest some ideas and perspectives for future studies. Design of the binary version as well as multi-objective version of TIMBO is an interesting topic for future investigations. Moreover, implementing TIMBO on various optimization problems and real-world optimization problems could achieve some significant contributions, as well.

\section{Conflicts of interest}

The authors declare no conflict of interest.

\section{Author contributions}

Conceptualization, F.A.Z.; methodology, M.D.; software, M.D.; validation, F.A.Z., O.P.M., and M.D.; formal analysis, O.P.M. and F.A.Z.; investigation, M.D., F.A.Z., and O.P.M.; resources, M.D.; data curation, F.A.Z.; writing — original draft preparation, M.D.; writing - review and editing, O.P.M. and F.A.Z.; visualization, M.D; supervision, M.D.; project administration, F.A.Z.; funding acquisition, O.P.M.

\section{References}

[1] M. Dehghani, Z. Montazeri, A. Dehghani, H. Samet, C. Sotelo, D. Sotelo, A. Ehsanifar, O. P. Malik, J. M. Guerrero, and G. Dhiman, "DM: Dehghani Method for modifying optimization algorithms", Applied Sciences, Vol. 10, No. 21, p. 7683, 2020.

[2] M. Dehghani, Z. Montazeri, A. Dehghani, O. P. Malik, R. Morales-Menendez, G. Dhiman, N. Nouri, A. Ehsanifar, J. M. Guerrero, and R. A. Ramirez-Mendoza, "Binary Spring Search Algorithm for Solving Various Optimization Problems", Applied Sciences, Vol. 11, No. 3, p. 1286, 2021.

[3] H. Givi, M. Dehghani, Z. Montazeri, R. Morales-Menendez, R. A. Ramirez-Mendoza, and N. Nouri, "GBUO: "The Good, the Bad, and the Ugly" Optimizer", Applied Sciences, Vol. 11, No. 5, p. 2042, 2021.

[4] S. A. Doumari, H. Givi, M. Dehghani, Z. Montazeri, V. Leiva, and J. M. Guerrero, "A New Two-Stage Algorithm for Solving Optimization Problems", Entropy, Vol. 23, No. 4, p. 491, 2021.

[5] S. A. Doumari, H. Givi, M. Dehghani, and O. P. Malik, "Ring Toss Game-Based Optimization Algorithm for Solving Various Optimization Problems", International Journal of Intelligent 
Engineering and Systems, Vol. 14, No. 3, pp. 545-554, 2021.

[6] A. Bose, T. Biswas, and P. Kuila, "A novel genetic algorithm based scheduling for multicore systems", Smart Innovations in Communication and Computational Sciences, pp. 45-54: Springer, 2019.

[7] J. Kennedy and R. Eberhart, "Particle swarm optimization", In: Proc. of ICNN'95international conference on neural networks, Vol. 4, ed: IEEE, pp. 1942-1948, 1995.

[8] E. Rashedi, H. Nezamabadi-Pour, and S. Saryazdi, "GSA: a gravitational search algorithm", Information sciences, Vol. 179, No. 13, pp. 2232-2248, 2009.

[9] R. V. Rao, V. J. Savsani, and D. Vakharia, "Teaching-learning-based optimization: a novel method for constrained mechanical design optimization problems", Computer-Aided Design, Vol. 43, No. 3, pp. 303-315, 2011.

[10] S. Mirjalili, S. M. Mirjalili, and A. Lewis, "Grey wolf optimizer", Advances in Engineering Software, Vol. 69, pp. 46-61, 2014.

[11] S. Saremi, S. Mirjalili, and A. Lewis, "Grasshopper optimisation algorithm: theory and application", Advances in Engineering Software, Vol. 105, pp. 30-47, 2017.

[12] M. Dehghani, Z. Montazeri, S. Saremi, A. Dehghani, O. P. Malik, K. Al-Haddad, and J. M. Guerrero, "HOGO: Hide Objects Game Optimization", International Journal of Intelligent Engineering and Systems, Vol. 13, No. 4, pp. 216-225, 2020.

[13] H. Karami, M. V. Anaraki, S. Farzin, and S. Mirjalili, "Flow Direction Algorithm (FDA): A Novel Optimization Approach for Solving Optimization Problems", Computers \& Industrial Engineering, Vol. 156, p. 107224, 2021. 